\title{
Comments on "Noise-Induced Transitions in a Simplified Model of the Thermohaline Circulation"
}

\author{
Adam Hugh Monahan \\ Institut für Mathematik, Humboldt-Universität zu Berlin, Berlin, Germany \\ AXEL TIMMERMANN \\ Institut für Meereskunde, Universität Kiel, Kiel, Germany \\ GERRIT LOHMANN \\ Geoscience Department, Bremen University, Bremen, Germany
}

20 November 2000 and 5 July 2001

Starting from the classical Stommel (1961) two-box model for the North Atlantic meridional overturning circulation, Timmermann and Lohmann (2000, hereafter TL) consider the dynamics of the following system of equations:

$$
\begin{aligned}
& \frac{d}{d t} y=-|1-y| y+\mu_{0}+y \epsilon \\
& \frac{d}{d t} \epsilon=-\frac{\epsilon}{\tau}+\frac{\sigma}{\tau} \xi,
\end{aligned}
$$

where $y$ represents the scaled salinity difference between the two boxes, $\mu_{0}$ is the scaled salinity forcing, $\xi$ is a white noise process, and $\epsilon$ is an Ornstein-Uhlenbeck (red noise) process with variance $\sigma^{2} / 2 \tau$ and autocorrelation $e$-folding time $\tau$. Timmermann and Lohmann associate $\epsilon$ with fluctuations of the temperature difference between the two boxes around the mean value (which is 1 in the scaled variables). The representation of variability in the temperature gradient by a red noise process is motivated by the findings of Lohmann and Schneider (1999) that in the Stommel model temperature differences vary on a much shorter timescale than salinity differences. As $\tau \rightarrow 0$, $\epsilon$ becomes Gaussian white noise, and the probability density function (PDF) of $y$ satisfies a one-dimensional Fokker-Planck equation (FPE) from which the stationary PDF (i.e., the PDF describing the system after the initial transients have died off) may be evaluated analytically. Because $\epsilon$ is multiplied by $y$ in Eq. (1), the $\tau \rightarrow 0$ limit must be taken

\footnotetext{
Corresponding author address: Dr. Adam Hugh Monahan, School of Earth and Ocean Sciences, University of Victoria, P.O. Box 3055 STN CSC, Victoria, BC V8P 5C2, Canada.

E-mail: monahana@uvic.ca
}

carefully. In technical jargon, Eq. (1) converges to a Stratonovich stochastic differential equation as $\tau \rightarrow 0$; a brief review of stochastic calculus and the FokkerPlanck equation is given in Penland (1996), and a more comprehensive discussion appears in Gardiner (1997). For $\tau \neq 0$, the PDF of $y$ alone is no longer described by an FPE. One can, however, write down a FPE for the joint PDF of $y$ and $\epsilon$. Unfortunately, the stationary version of this FPE is a partial differential equation in two variables that cannot be solved analytically. In this note, we comment on two aspects of TL: first, the derivation and interpretation of Eqs. (1) and (2), and second, the validity of the approximation used in TL to obtain analytic forms for the stationary PDF of $y$ for $\tau$ $\neq 0$.

We first comment on the derivation and interpretation of Eqs. (1) and (2). The original system of equations in TL describes the dynamics of the salinity gradient, $\Delta S$, when the temperature gradient $\Delta T$ is described as red noise fluctuations $\Delta T^{\prime}$ around a mean value $\Delta T_{0}$ :

$$
\begin{aligned}
\frac{d}{d t^{*}} \Delta S= & -\frac{c}{V}\left|\alpha \Delta T_{0}+\alpha \Delta T^{\prime}-\beta \Delta S\right| \Delta S \\
& +\frac{S_{0}}{h}(P-E) \\
\frac{d}{d t^{*}} \Delta T^{\prime}= & -\frac{1}{\tau^{*}} \Delta T^{\prime}+\frac{\Sigma}{\tau^{*}} \xi,
\end{aligned}
$$

where $t^{*}$ is the dimensional time, $V$ is the box volume, $c$ is the proportionality constant between the density gradient and the meridional flux, $\alpha$ and $\beta$ are respectively the thermal and haline expansion coefficients, $S_{0}$ is a reference salinity, $h$ is the box depth, $P-E$ is the salinity forcing, $\tau^{*}$ is the temperature fluctuation relax- 

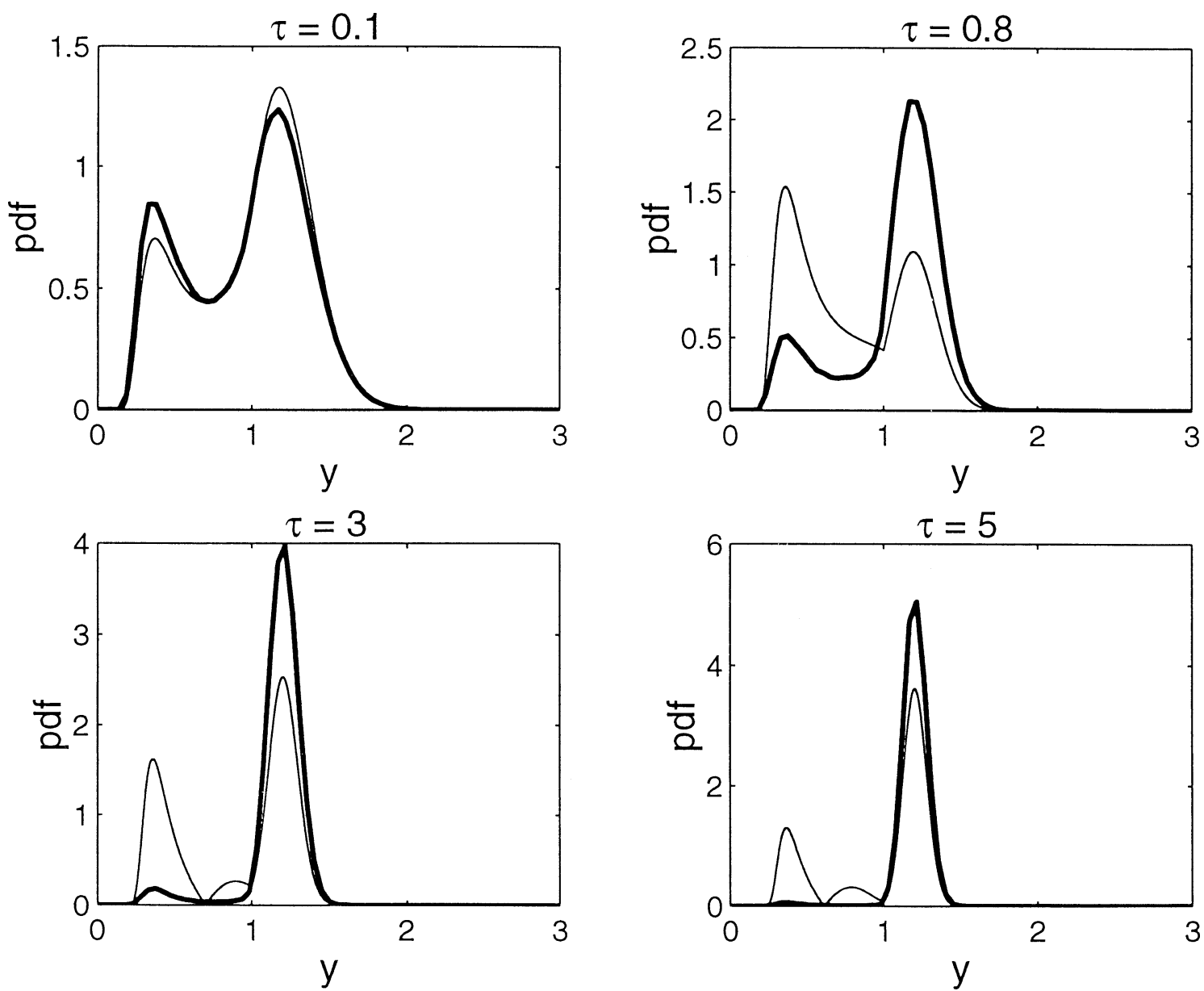

FIG. 1. Stationary PDF of $y$ from numerical integration (thick line) and from the UCNA approximation (thin line) for $\sigma=0.3$.

ation timescale, $\Sigma$ is a noise strength, and $\xi$ is Gaussian white noise. Timmermann and Lohmann argue that Eqs. (1) and (2) follow from (3) and (4) under a suitable rescaling of variables. However, in Eq. (3) the process $\Delta T^{\prime}$ appears only inside the absolute value sign, while in Eq. (1) the process appears outside the absolute value sign. In fact, Eq. (1) does not follow from (3). Furthermore, it can be shown that for (3) the stationary PDF of $\Delta S$ in the limit that $\Delta T^{\prime}$ becomes a white noise process is a delta function at $\Delta S=0$ (P. Imkeller 2000, personal communication), which is not physically reasonable. For $\tau \neq 0$, Eqs. (3) and (4) define a meaningful stochastic differential equation. However, as this system must be solved numerically, no advantage has been gained in representing $\Delta T$ as a red noise process; the analysis of the stochastically forced original Stommel model is no more complicated.

An alternate interpretation of (1) is as follows. We consider a generalization of the Stommel (1961) model:

$$
\begin{aligned}
\frac{d}{d t^{*}} \Delta S= & \left(-\frac{c}{V}|\alpha \Delta T-\beta \Delta S|+\eta\right) \Delta S \\
& +\frac{S_{0}}{h}(P-E) \\
\frac{d}{d t^{*}} \Delta T= & \left(-\frac{c}{V}|\alpha \Delta T-\beta \Delta S|+\eta\right) \Delta T+\frac{F_{o a}}{C_{p}},
\end{aligned}
$$

where $C_{p}$ is the oceanic heat capacity. The freshwater forcing $P-E$ is an unspecified function of $\Delta T$, and the atmosphere-ocean heat flux $F_{o a}$ has the net effect of relaxing $\Delta T$ to some value $\Delta T_{0}$. The quantity $\eta$ in Eqs. (5) and (6) is a parameterization of the eddy transport of temperature and salinity between the boxes; this eddy mixing may be associated with transport due to, for example, the wind-driven gyres or quasigeostrophic eddies. The process $\eta$ is not constrained to be positive, so eddy transport between the boxes may be upgradient (see, e.g., Nakamura and Chao 2000). A similar term 

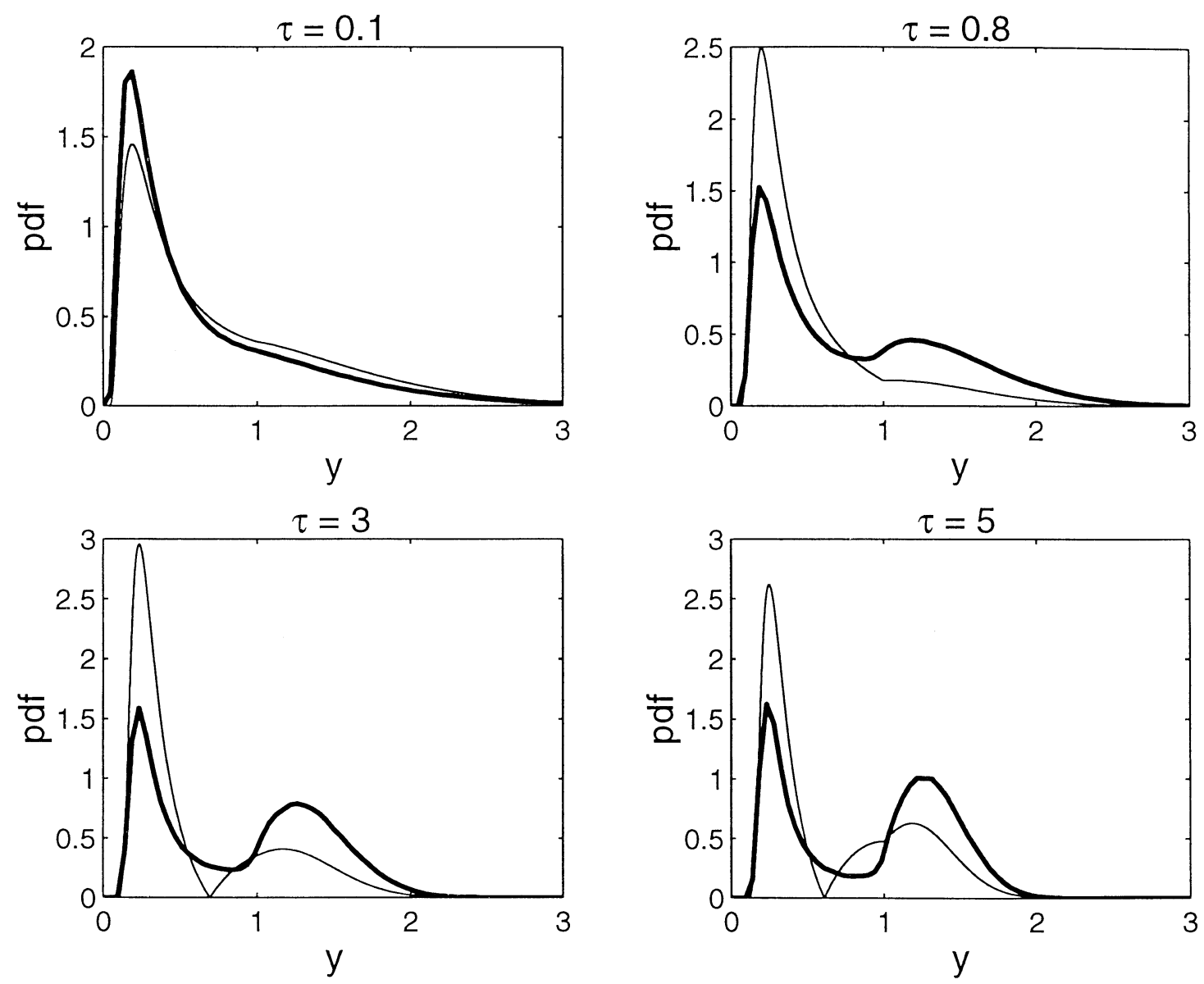

FIG. 2. As in Fig. 1 but for $\sigma=1$.

appears in response to fluctuations in mechanical forcing in the model of the thermally and wind-driven ocean circulation introduced by Maas (1994). In general, $\eta$ should have a nonzero mean value, so the eddy transport is on average downgradient. The goal here, however, is to describe a meaningful interpretation of the model analyzed in TL in which $\eta$ is of mean zero.

As in Cessi (1994), we assume that the timescale on which $\Delta T$ is relaxed to $\Delta T_{0}$ by the thermal forcing $F_{o a}$ is sufficiently small, relative to the timescales of salinity dynamics, that $\Delta T \simeq \Delta T_{0}$. Further, we model $\eta$ as a red noise (Ornstein-Uhlenbeck) process with autocorrelation $e$-folding timescale $\tau^{*}$ and variance $\Sigma^{2} / 2 \tau^{*}$. Then we obtain the system

$$
\begin{aligned}
\frac{d}{d t^{*}} \Delta S= & -\frac{c}{V}\left|\alpha \Delta T_{0}-\beta \Delta S\right| \Delta S+\eta \Delta S \\
& +\frac{S_{0}}{h}(P-E) \\
\frac{d}{d t^{*}} \eta= & -\frac{\eta}{\tau^{*}}+\frac{\Sigma}{\tau^{*}} \xi\left(t^{*}\right),
\end{aligned}
$$

where $\xi\left(t^{*}\right)$ is a white noise process. Defining the nondimensional quantities

$$
\begin{aligned}
& t=\frac{c \alpha \Delta T_{0}}{V} t^{*} \\
& y=\frac{\beta}{\alpha \Delta T_{0}} \Delta S \\
& \epsilon=\frac{V}{c \alpha \Delta T_{0}} \eta,
\end{aligned}
$$

Eqs. (7) and (8) reduce to (1) and (2) where

$$
\begin{aligned}
\mu & =\frac{\beta V S_{0}}{c h\left(\alpha \Delta T_{0}\right)^{2}}(P-E) \\
\tau & =\frac{c \alpha \Delta T_{0}}{V} \tau^{*} \\
\sigma & =\left(\frac{V}{c \alpha \Delta T_{0}}\right)^{1 / 2} \Sigma .
\end{aligned}
$$

In the above, we have used the fact that for a white noise process,

$$
\xi\left(t^{*}\right)=\left(\frac{t}{t^{*}}\right)^{1 / 2} \xi(t)
$$


under a rescaling of time, which follows from the fact that the white noise must be delta-correlated in both the dimensional and nondimensional variables.

Thus, a meaningful physical interpretation can be made of Eqs. (1) and (2), but this interpretation differs from that of TL.

For $\tau \neq 0$, the stationary FPE of the system (1)-(2) does not yield an analytic solution. To circumvent this problem, TL employ an approximation due to Jung and Hänggi (1987), known as the "Unified Coloured Noise Approximation" (UCNA), to reduce the system (1)-(2) to an approximate one-dimensional system, whose associated stationary Fokker-Planck equation admits an analytic solution. Timmermann and Lohmann calculate the following UCNA expression for the stationary PDF of $y$ :

$$
\begin{array}{ll}
p_{s}(y, \tau)=N_{1} y^{-1}\left|1+\tau\left(\frac{\mu_{0}}{y}-y\right)\right| \exp \left\{\frac{2}{\sigma^{2}}\left[-\frac{1}{2} \tau\left(-1+y+\frac{\mu_{0}}{y}\right)^{2}-\ln y+y-\frac{\mu_{0}}{y}\right]\right\}, & 0<y \leq 1 \\
p_{s}(y, \tau)=N_{2} y^{-1}\left|1+\tau\left(\frac{\mu_{0}}{y}+y\right)\right| \exp \left\{\frac{2}{\sigma^{2}}\left[-\frac{1}{2} \tau\left(1-y+\frac{\mu_{0}}{y}\right)^{2}+\ln y-y-\frac{\mu_{0}}{y}\right]\right\}, & y>1,
\end{array}
$$

where $N_{1}, N_{2}$ are appropriate normalizations. Figures 6 and 7 of TL plot $p_{s}$ as a function of $y$ for different values of $\tau$ and $\sigma$. For small values of $\tau$, the results resemble those of the white noise limit. For increasing $\tau, p_{s}$ given by (16) displays qualitatively different behavior. In particular, nodes and new extrema appear in the PDF. These results are interpreted as "noise-induced transitions," and the analogy of quantum-mechanical tunneling is used to describe the passage of the system across the node of the PDF. We now comment on the validity of the UCNA in the analysis of TL.

Another approach to determine an approximation of the PDF associated with (1)-(2) is to integrate the equations numerically; the simplest algorithm is a forward Euler discretization (Kloeden and Platen 1992). Denoting the discrete time step by $\delta$ so that

$$
t_{k}=k \delta \text {, }
$$

the forward Euler discretization of (1)-(2) is

$$
\begin{aligned}
& y_{t_{k}}=y_{t_{k-1}}+\delta\left(-\left|1-y_{t_{k-1}}\right| y_{t_{k-1}}+\mu_{0}+y_{t_{k-1}} \epsilon_{t_{k-1}}\right) \\
& \epsilon_{t_{k}}=\epsilon_{t_{k-1}}-\delta \frac{\epsilon_{t_{k-1}}}{\tau}+\sqrt{\delta} \frac{\sigma}{\tau} W_{t_{k-1}},
\end{aligned}
$$

where $W_{t_{k}}$ is a sequence of zero-mean, unit variance Gaussian random variables. Equations (18) and (19) were integrated for 150000 time units, with a time step $\delta=0.05$ for the parameter values $\tau, \sigma$ used in Figs. 6 and 7 of TL. Figures 1 and 2 display Gaussian kernel density estimates of the PDFs obtained from the simulation, along with the UCNA approximations (16). The numerical results are robust to reduction of the stepsize $\delta$, and inspection of the time series indicates that the record is long enough for estimation of the PDF from the time series to be appropriate.

Comparing the numerical and UCNA results in Figs. 1 and 2, it is clear that for small values of $\tau$, the stationary PDFs produced by numerical integration and by the UCNA are in close agreement. However, for $\tau \sim$ $O(1)$, there are marked differences. In particular, the PDFs produced by numerical integration do not display any nodes or new extrema. Instead, the result of raising $\tau$ for fixed $\sigma$ is seen to be a shift of the PDF toward the right-hand peak. The differences between the numerical and UCNA approximations occur because of a breakdown of the validity of the UCNA for $\tau$ of $O(1)$.

By construction, the UCNA assumes a timescale separation between the processes $y$ and $\epsilon$; it is only valid when $\epsilon$ varies much more rapidly than $y$. This implies that the UCNA is a small $\tau$ approximation, but how small is "small?" Timmermann and Lohmann note that the domain of validity of the UCNA is given by the following pair of inequalities:

$$
\begin{gathered}
\kappa(y, \tau)=\frac{1}{\sqrt{\tau}}-\sqrt{\tau}\left(y-\frac{\mu_{0}}{y}\right) \gg \tilde{t}^{-1}, \\
0<y \leq 1 \\
\kappa(y, \tau)=\frac{1}{\sqrt{\tau}}-\sqrt{\tau}\left(-y-\frac{\mu_{0}}{y}\right) \gg \tilde{t}^{-1}, \\
y>1,
\end{gathered}
$$

where $\tilde{t}$ is a "characteristic timescale" of variability of $y$. Defining a "typical" value of $y$, TL argue for the global (in y) satisfaction of these conditions. In fact, their validity must be considered locally in $y$. In particular, the UCNA certainly fails wherever $\kappa$ vanishes. For $0<y \leq 1$, this occurs for

$$
y=\frac{1}{2 \tau}+\sqrt{\left(\frac{1}{2 \tau}\right)^{2}+\mu_{0}}
$$

(a second root is discarded because it occurs for $y<0$, outside the domain of consideration). For $\tau<1 /(1-$ $\left.\mu_{0}\right)$, this root also falls outside of $[0,1]$. However, for $\tau \geq 1 /\left(1-\mu_{0}\right), \kappa$ vanishes for $y \in[0,1]$, and the 
UCNA fails within the domain of interest. In particular, it is clear from (16) that $\kappa$ vanishes at precisely the values $y$ where $p_{s}$ has a node. Thus, the emergence of zeros in the PDF of $y$ is an artifact of the breakdown of the UCNA. In fact, the UCNA fails not just at the points at the zeros of $\kappa$, but in a surrounding neighborhood, as is demonstrated by the differences between the numerical and UCNA PDFs for $\tau=0.8<1 /(1-$ $\left.\mu_{0}\right)$. For $y>1, \kappa$ never vanishes, but because the overall amplitude of the PDF is a function of its global structure, at best only the shape of the PDF for $y>1$ will agree with that produced by the UCNA. Thus, for $\tau$ of $O(1)$, the UCNA breaks down locally in $y$, with global consequences for the structure of the PDF.

Calculating the stationary PDF of a system in a onedimensional potential subject to colored noise remains an unsolved problem in physics. A number of different approximations have been proposed, but they are valid only in the limit of small or of very large $\tau$ (Horsthemke and Lefever 1984; Hänggi and Jung 1995). To obtain the stationary PDFs of $y$ in the case where its timescale is of the same order of magnitude as $\epsilon$, at present we must take recourse to numerical methods. We note that an essential conclusion of TL is unchanged, namely that increasing $\sigma$ populates the left-hand peak of the stationary PDF of $y$ at the expense of the right-hand peak, while increasing $\tau$ has the opposite effect.

Overall the idea is supported that changing noise characteristics might have a significant effect on the climatic mean states and their stability; this point is discussed in a more general framework in Palmer (2001). The nonlinear paradigm of noise-induced transitions is consistent with the findings recently reported by Aeberhardt et al. (2000) using a stochastically forced model of intermediate complexity. It might be interesting to study how the effect of noise-induced transitions of the THC translates to other more complex models.

\section{REFERENCES}

Aeberhardt, M., M. Blatter, and T. F. Stocker, 2000: Variability on the century time scale and regime changes in a stochastically forced zonally averaged ocean-atmosphere model. Geophys. Res. Lett., 27, 1303-1306.

Cessi, P., 1994: A simple box model of stochastically forced thermohaline flow. J. Phys. Oceanogr, 24, 1911-1920.

Gardiner, C. W., 1997: Handbook of Stochastic Methods for Physics, Chemistry and the Natural Sciences. Springer, $442 \mathrm{pp}$.

Hänggi, P., and P. Jung, 1995: Colored noise in dynamical systems. Adv. Chem. Phys., 89, 239-326.

Horsthemke, W., and R. Lefever, 1984: Noise-Induced Transitions: Theory and Applications in Physics, Chemistry, and Biology. Springer, $318 \mathrm{pp}$.

Jung, P., and P. Hänggi, 1987: Dynamical systems: A unified colourednoise approximation. Phys. Rev., A35, 4464-4466.

Kloeden, P. E., and E. Platen, 1992: Numerical Solution of Stochastic Differential Equations. Springer, 632 pp.

Lohmann, G., and J. Schneider, 1999: Dynamics and predictability of Stommel's box model: A phase space perspective with implications for decadal climate variability. Tellus, 51A, 326-336.

Maas, L. R. M., 1994: A simple model for the three-dimensional, thermally and wind-driven ocean circulation. Tellus, 46A, 671680 .

Nakamura, M., and Y. Chao, 2000: On the eddy isopycnal thickness diffusivity of the Gent-McWilliams subgrid mixing parameterization. J. Climate, 13, 502-510.

Palmer, T. N., 2001: A nonlinear dynamical perspective on model error: A proposal for nonlocal stochastic-dynamic parameterisation in weather and climate prediction models. Quart. J. Roy. Meteor. Soc., 127, 279-304.

Penland, C., 1996: A stochastic model of IndoPacific sea surface temperature anomalies. Physica D, 98, 534-558.

Stommel, H. M., 1961: Thermohaline convection with two stable regimes of flow. Tellus, 13, 224-230.

Timmermann, A., and G. Lohmann, 2000: Noise-induced transitions in a simplified model of the thermohaline circulation. J. Phys. Oceanogr., 30, 1891-1900. 